Int. J. Morphol.,

35(1):363-367, 2017

\title{
Hígado: el segmento portal V
}

\author{
Liver: the portal segment $\mathbf{V}$
}

César Augusto Durand López

DURAND, L. C. A. Hígado: el segmento portal V. Int. J.Morphol., 35(1):363-367, 2017.

RESUMEN: Algunos autores han propuesto la existencia de un número variable de segmentos portales hepáticos (A05.8.01.033)*. Sin embargo, estas diferencias radican en la manera de describir el segmento medial derecho+ (segmento V), lo cual ha provocado confusión al estudiar la anatomía del hígado (A05.8.01.001). Para explicar la correcta anatomía del segmento portal V (A05.8.01.047), estudiamos la estructura vascular intrahepática de 200 hígados humanos de individuos de ambos sexos, de diferentes edades, desde fetos hasta octogenarios. Ciento cincuenta hígados fueron inyectados con acrílico a través de la vena porta hepática (A12.3.12.001), arteria hepática propia (A12.2.12.029), conducto hepático común (A05.8.01.061), vena cava inferior (A12.3.09.001) y venas hepáticas (A12.3.09.005). Los 50 hígados restantes fueron disecados en fresco, basándonos en los moldes acrílicos obtenidos previamente. Se encontró solo una rama portal segmentaria para la división medial derecha (A05.8.01.046) entre la fisura portal derecha (A05.8.01.036) y la fisura portal principal (A05.8.01.035). Esta rama fue la más voluminosa ( $10 \mathrm{~mm}$ de diámetro en su base) entre las siete ramas portales segmentarias + encontradas. La misma, luego de penetrar $2 \mathrm{~cm}$ en el parénquima en dirección a la porción superior del hígado (A05.8.01.003), dio ramas anteriores, laterales, posteriores y mediales, que se distribuyeron desde la placa hiliar+ hasta la cúpula hepática, desde la fisura portal derecha hasta la fisura portal principal, desde el margen inferior (A05.8.01.024) hasta la vena cava inferior por detrás. En $80 \%$ de los hígados, esta rama nació de la vena porta derecha (A12.3.12.002), y en $20 \%$ de la vena porta izquierda (A12.3.12.005); en estos casos el lóbulo izquierdo (A05.8.01.027) era más voluminoso que el derecho (A05.8.01.026). Estos hallazgos llevan a plantear nuevas definiciones y conceptos, los que se proponen en la discusión.

PALABRAS CLAVE: Segmento portal V; Vena porta izquierda; Vena porta derecha.

\section{INTRODUCCIÓN}

El hígado es un órgano sumamente vascularizado. Sin embargo, en la literatura solo contamos con descripciones esquemáticas de la anatomía intrahepática basadas en teorías que actualmente confunden a radiólogos y cirujanos (Terminology Committee of the IHPBA, 2000). Diferentes autores han proclamado su propia segmentación portal, siendo el segmento $\mathrm{V}$ el que mayor confusión ha generado. Este estudio pretende contribuir a aclarar la anatomía de este segmento.

\section{MATERIAL Y MÉTODO}

Se estudiaron 200 hígados humanos de ambos sexos, desde fetos hasta octogenarios. De éstos hígados, 150 de ellos fueron inyectados con acrílico líquido-sólido más colorantes, a través de la vena porta, la arteria hepática, las vías biliares, la vena cava inferior y las venas hepáticas. Los restantes 50 hígados, fueron disecados en fresco basados en los hallazgos obtenidos de los hígados inyectados.
Usamos la Terminología Anatómica Internacional. Colocamos el número identificatorio cuando citamos un elemento anatómico por primera vez en el texto, cerramos entre paréntesis los elementos inconstantes y marcamos con una + aquellos no catalogados en la Terminologia Anatomica Internacional.

\section{RESULTADOS}

De los 200 hígados estudiados, en 160 (80\%) la rama portal para el segmento $\mathrm{V}+$ nació en el margen superior de la rama portal derecha, a $1 \mathrm{~cm}$ de la división de la vena porta. En 40 casos $(20 \%)$ se originó de la vena porta izquierda (Fig. 1), en estos casos, la rama portal izquierda se profundizó superiormente en el parénquima e hizo una curva de convexidad superior derecha, de donde se originó la rama V, para luego continuar su trayecto horizontal; esto sucedió a $1 \mathrm{~cm}$ de la bifurcación portal.

En todos los casos, la rama segmentaria V se elevó dirigiéndose a la cúpula hepática tratando de colocarse sobre el margen derecho de la vesícula biliar. Una vez que 
sobrepasó el plano de las venas hepáticas, esta voluminosa rama emitió en forma alternada, y una por una, dos o más ramas anteriores, dos o más ramas laterales, dos o más ramas posteriores y dos o más ramas mediales, siendo estas últimas las que llegaron más alto. Estas ramas dispuestas en todas direcciones, abarcaron el territorio comprendido entre la fisura portal derecha (cuyo plano se inclinó 60 grados hacia la derecha en relación al plano sagital del hígado) y la fisura portal principal (cuyo plano se inclinó 30 grados hacia la izquierda en relación al plano sagital del hígado), desde el margen anterior hepático hasta la vena cava inferior por detrás (Fig. 2). Este gran territorio abarcado explica el mayor grosor de esta rama portal (10 $\mathrm{mm}$ de diámetro en su origen). Así se observa que, en una región comprendida desde una delgada franja parenquimal (correspondiente a un punto entre el margen derecho de la vesícula biliar y el ángulo anterior derecho del hígado), hasta la mitad de la fosa cística,

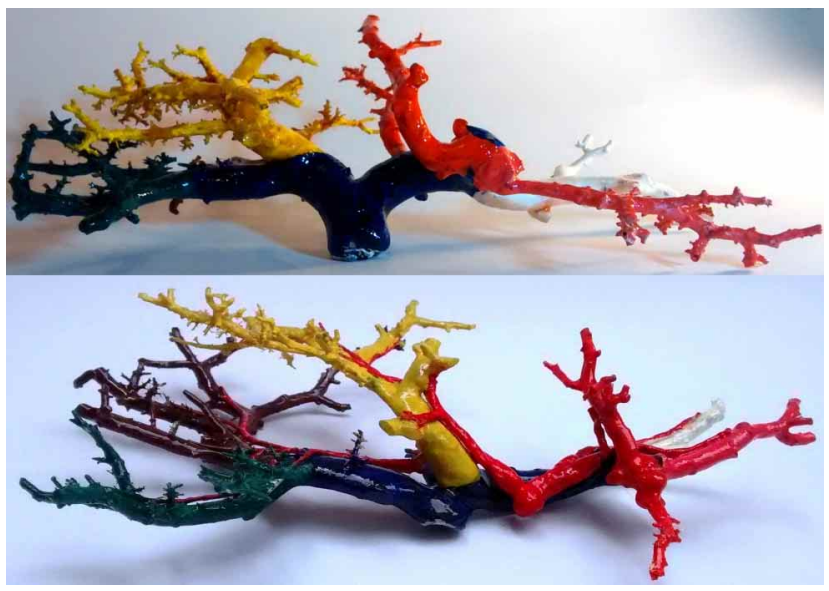

Fig. 1. Front view of two portal acrylic livers. Upper, segmental branch V (yellow) arises from the right portal vein; at the lower branches off the left.

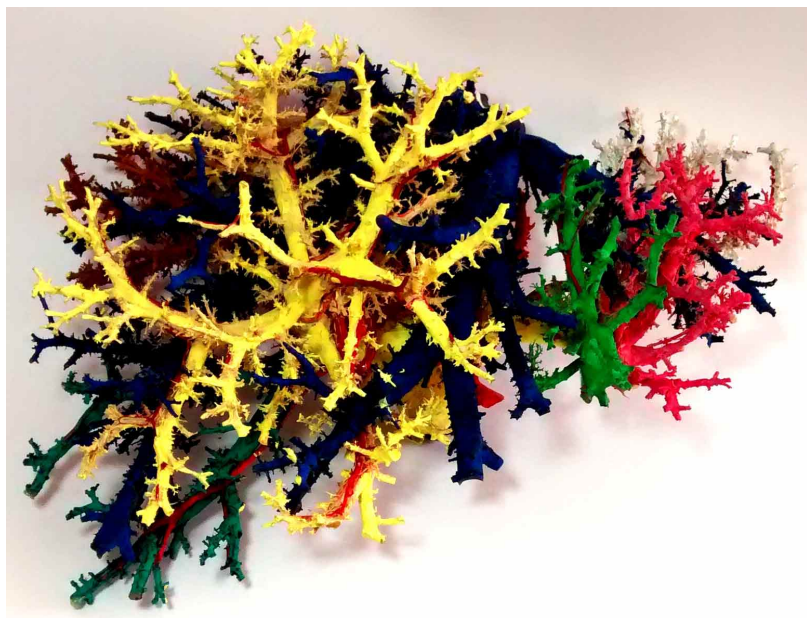

Fig. 2 Front view of portal acrylic, segment $\mathrm{V}$ tributaries in yellow. We see anterior, lateral, posterior and medial branches, distributed between intermediate hepatic and right veins, from the inferior edge B64to the inferior vena cava. y desde el margen inferior del hígado hasta la placa hiliar+, el voluminoso segmento $\mathrm{V}$ se va abriendo como un cono invertido, cuyas paredes divergen 90 grados, hasta abarcar la superficie antes citada (entre la vena hepática derecha y la vena intermedia), disponiéndose su porción posterior sobre la mitad derecha del segmento I (lóbulo caudado) (A05.8.01.044) (Fig. 3).

Hemos encontrado la rama portal segmentaria $\mathrm{V}$ como una de las 7 ramas segmentarias portales abordables a nivel de la placa portal+ en el hilio hepático inferior+. No hemos encontrado ninguna rama portal que pueda justificar la existencia de un octavo segmento (A05.8.01.048).

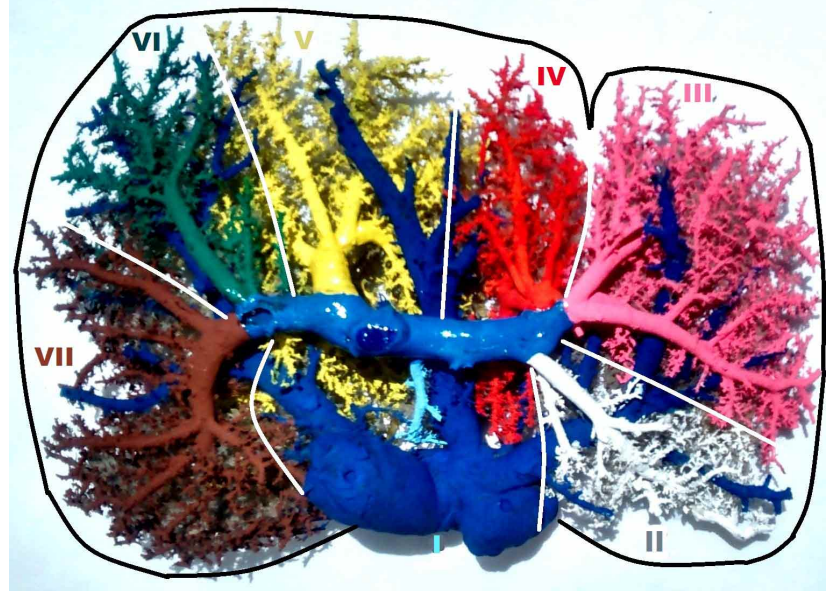

Fig. 3. Inferior view of portal acrylic (colors), cava and tributaries (blue). The branch V (yellow) rises above giving anterior, lateral, posterior and medial branches. Segment V posterior branches are distributed among the portal vein, the intermediate and right hepatic veins above segment I (light blue).

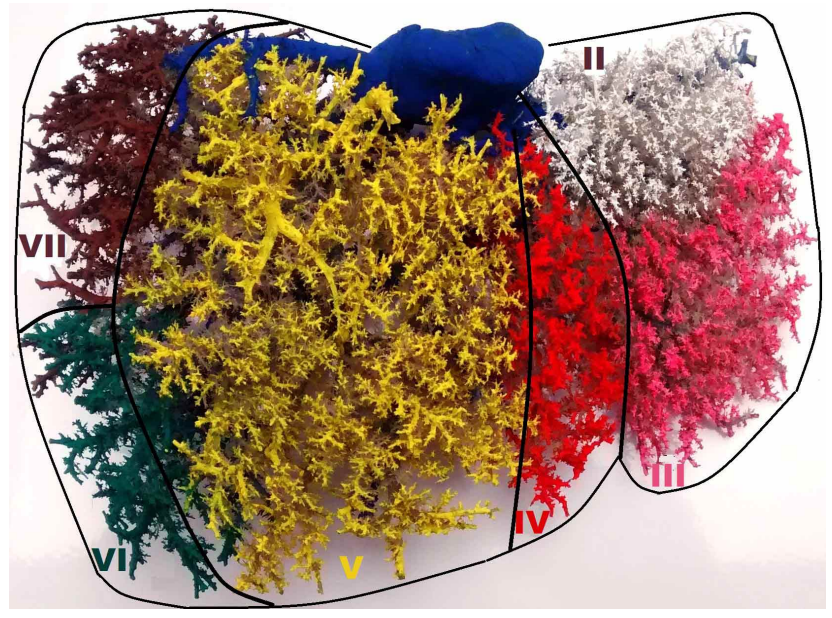

Fig. 4. Front view of portal-cava acrylic. The segment V (yellow) limits ahead with the inferior border of the liver, behind the inferior vena cava, medially with the main portal fissure containing the intermediate hepatic vein and the medial edge of the segment IV, laterally with the right fissure containing the right hepatic veins and the medial edge of the segments VI and VII. 
La irrigación arterial para el segmento $\mathrm{V}$ nació de la rama derecha de la arteria hepática propia (A12.2.12.030) en $94 \%$ de casos, de la rama izquierda de la arteria hepática propia (A12.2.12.035) en un $1 \%$ y de una rama de la arteria mesentérica superior (A12.2.12.053), anastomosada a la arteria hepática propia, en el $5 \%$. El drenaje biliar del segmento $\mathrm{V}$ se realizó en el conducto hepático derecho (A05.8.01.062) junto con los segmentos VI y VII en $71 \%$ de casos. Se dispuso como único formante del conducto hepático derecho en el $29 \%$ de los casos. Esto debido a que el tronco VI-VII drenó en el conducto hepático izquierdo (A05.8.01.065) en el $21 \%$, y por lo tanto este drenaje nunca fue más allá de $1 \mathrm{~cm}$ de distancia de la formación del conducto hepático común; drenó también en el hepático común en $6 \%$ y en el conducto cístico (A05.8.02.011) en $2 \%$.

El drenaje venoso del segmento $\mathrm{V}$ se realizó tanto en la vena hepática intermedia (A12.3.09.007) como en la vena hepática derecha (A12.3.09.006).

Encontramos tres amplias fisuras portales verticales [la fisura derecha (A05.8.01.036), la principal (A05.8.01.035) y la umbilical (A05.8.01.034)] además de tres delgadas fisuras horizontales+, una entre los segmentos II y III, otra entre los segmentos VI y VII, la última entre la cara superior del segmento I y la cara inferior y posterior de los segmentos IV y V.

Por otro lado, el plano que dividió el hemihígado derecho del hemihígado izquierdo varió según el lugar de emergencia de la rama segmentaria V. Cuando la rama portal V nació de la vena porta derecha $(80 \%)$, el plano de la fisura portal principal dividió el lóbulo derecho del izquierdo, esto fue, un plano inclinado 30 grados hacia la izquierda con relación al plano sagital del hígado, con un trayecto lineal recto que va desde el centro de la fosa vesicular hasta el margen izquierdo de la cava inferior.

Cuando la rama portal segmentaria $V$ nació de la vena porta izquierda, el plano divisor entre ambos hemi-hígados fue el de la fisura portal derecha, inclinado 60 grados hacia la derecha en relación al plano sagital hepático, este siguió una línea curva de convexidad derecha que fue desde un punto equidistante entre el margen vesicular derecho y el ángulo hepático antero-inferior derecho, hasta el margen derecho de la cava inferior, de tal manera que en este último caso (20\%) el lóbulo izquierdo fue más grande que el derecho.

\section{DISCUSIÓN}

Distintos autores han dado diferentes cifras cuando se ha tratado del número de ramas segmentarias portales al describir la segmentación del hígado. Cantlie (1898) fue el primero que mencionó la correcta división entre los lóbulos derecho e izquierdo del hígado por una línea que se extiende desde la fosita vesicular hasta el margen izquierdo de la vena cava inferior. Hjortsjo (1951) describió la oblicuidad de la fisura del hígado y fue el primero en demostrar la distribución segmentaria de las ramas de la arteria hepática, conductos biliares y vena porta. Asimismo, Hjortsjo describió cinco segmentos hepáticos, Healey et al. (1953) y Healey \& Schroy (1953) describieron 9, Couinaud $(1954,1989)$ describió 8, Hidayet et al. (1977) describió 9.

El concepto de segmento portal se refiere aquella porción de parénquima hepático irrigado por una rama terminal de la vena porta (segunda división portal), acompañada de una rama terminal de la arteria hepática propia o su reemplazante y un conducto de drenaje biliar; estos vasos, por ser terminales, no se anastomosan con los vasos de segmentos vecinos, creando fisuras intersegmentarias. De tal manera que el segmento posee ya dos características definitorias: la primera, poseer un pedículo vascular terminal; la segunda, estar limitado por fisuras.

Las ramas primarias de la vena porta (izquierda y derecha) dan las 7 ramas portales segmentarias (ramas secundarias y terminales) dentro de la estrecha placa portal+ del hilio inferior; a su vez, los pedículos al expandirse dentro del parénquima de manera centrífuga, dan forma cónica a los segmentos, con el vértice apuntando al pedículo hepático inferior.

La rama portal segmentaria $\mathrm{V}$ es la más gruesa y caudalosa de las 7 ramas segmentarias portales, penetra en el parénquima colocándose a la derecha de la vesícula biliar, en dirección del cenit hepático, y luego de elevarse $2 \mathrm{~cm}$ comienza a dar en forma alternada, una por una, dos o más ramas anteriores, dos o más laterales, dos o más posteriores y dos o más mediales, siendo estas últimas las que llegan más alto en la cúpula hepática. Además, las ramas del tallo segmentario $\mathrm{V}$ se distribuyen desde el pedículo portal desde inferior, hasta la cúpula hepática hacia superior, desde la fisura portal derecha hasta la fisura portal principal, desde el margen anterior del hígado hasta la vena cava inferior hacia posterior. Esta extensión parenquimal explica lo caudaloso de esta rama portal segmentaria (Fig. 4). La mitad posterior del segmento V (del hilio inferior hacia posterior) monta sobre la cara superior de la mitad derecha del segmento I (sobre la mitad izquierda se coloca la mitad posterior del segmento IV). La fisura portal principal pasa sobre la línea media del segmento I, de anterior hacia posterior, sin llegar a dividirlo, por estas razones la mitad posterior de los segmentos IV y V no se ven en la cara inferior del hígado, en sus lugares se ve al segmento I. 
Otro hallazgo importante lo constituye el nacimiento de la rama portal segmentaria $\mathrm{V}$ a partir de la vena porta derecha en el $80 \%$ de casos, determinando que los segmentos portales I, II, III y IV sean izquierdos y los segmentos V, VI y VII sean derechos. En $20 \%$ de casos, la rama segmentaria $\mathrm{V}$ nace de la vena porta izquierda, aquí los segmentos I, II, III, IV y V son izquierdos y los segmentos VI y VII son derechos.

Chaib et al . (2005) describieron la división de la vena porta en una rama derecha, otra izquierda y una rama intermedia, en $17 \%$ de 60 casos de hígados de adultos necropsiados. Lo que encontró este autor fue la gran rama portal para el segmento $\mathrm{V}$, naciendo de la vena porta izquierda. La idea de tripartición portal es errada, ya que esta rama portal "intermedia" es terminal y no da ramas segmentarias como si lo hace las ramas izquierda y derecha. Esta rama "intermedia" llega a distribuirse en el territorio segmentario V. Además, ciertamente esta rama portal intermedia se distribuyó en un territorio ubicado entre el segmento izquierdo IV y los derechos VI y VII, es decir, entre la fisura portal derecha y la fisura portal principal, llegando así a cumplir los requisitos que definen un segmento: tener un pedículo vascular terminal y estar separado del resto por fisuras, verticales en este caso. En nuestra opinión, en el estudio que comentamos, la rama $\mathrm{V}$ nació de la vena porta derecha en $83 \%$ de casos y de la vena porta izquierda en $17 \%$.

Fasel \& Schenk (2013) manifestaron la incongruencia de la división segmentaria de Couinaud con las modernas imágenes de la vasculatura hepática. Kimura et al . (2015) mencionaron que en Japón usan la clasificación de Healey \& Schroy combinada con la de Couinaud (1989) para describir la ubicación de los tumores en el hígado. Esta falta de definición anatómica recuerda la reunión del comité para la terminología anatómica de la Asociación Internacional de Cirugía Hepato-pancrato-biliar en Brisbane el año 2000, para tomar acuerdos respecto a la descripción anatómica de las hepatectomias: en relación al hígado no hay conceptos anatómicos claros.

Consideramos importante poder abordar los pedículos segmentarios a nivel de su nacimiento en la placa portal, para controlar el aporte sanguíneo de cualquier segmento, así también conocer que territorio parenquimal corresponde a cada pedículo segmentario. Esto nos lleva a considerar otro concepto: cuando la rama portal segmentaria $\mathrm{V}$ nace de la vena porta derecha, la fisura que divide el hemi hígado derecho del izquierdo es la fisura portal principal. Cuando la rama portal segmentaria $\mathrm{V}$ nace de la vena porta izquierda, es la fisura portal derecha la que divide ambos hemi hígados; en estos casos el hemi hígado izquierdo es más voluminoso que el derecho.
La irrigación arterial del segmento $\mathrm{V}$ puede provenir desde cualquier arteria abdominal cercana al hígado como describen Hiatt et al . (1994), Chanasong et al . (2014) y Bianchi et al . (2014), pero cualquiera sea su origen siempre tomará el camino de ingreso como pedículo segmentario. En nuestros casos la irrigación provino de la arteria hepática propia en $94 \%$, de la arteria mesentérica superior en $5 \%$ y de la arteria hepática izquierda en $1 \%$. Estas arterias se anastomosan en la placa hiliar.

El drenaje biliar del segmento $\mathrm{V}$ forma el conductillo más constante y caudaloso, formando con el VI y el VII el conducto biliar derecho en $71 \%$ y siendo el único formante en $29 \%$ de nuestros casos, consideramos que la clasificación del drenaje biliar por segmentos es más descriptiva (Tolino et al ., 2010).

El drenaje de retorno venoso se realiza en las venas derechas y en la vena intermedia principalmente; el drenaje directo en la cava inferior es mínimo (Mitidieri \& Loccisano, 2014).

No se altera el orden numérico de los segmentos si consideramos el segmento $\mathrm{V}$ como el único ubicado entre la fisura portal derecha y la fisura portal principal, naciendo su pedículo portal en la rama derecha o la izquierda (que sí afecta a la división segmentaria de Couinaud).

Llamaríamos al segmento $\mathrm{V}$ como segmento medial derecho en caso que el pedículo nazca de la vena porta derecha, sin embargo, si el pedículo nace en la vena porta izquierda el segmento se llamaría medial izquierdo, quitándole la denominación al segmento IV. Toda esta confusión se podría evitar si lo llamamos simplemente segmento V, sea su pedículo portal izquierdo o derecho (Figs. 1, 3 y 4).

En este trabajo demostramos que el segmento portal $V$ es el más grande y el único cuyo pedículo puede nacer en cualquiera de las dos ramas portales primarias.

La presencia de un único segmento portal (V) en la zona correspondiente a la división medial derecha nos permite proponer la segmentación hepática con siete segmentos portales, donde los segmentos I, II, III y IV siempre son izquierdos, y los segmentos VI y VII siempre son derechos, el segmento $\mathrm{V}$ puede tener su pedículo vascular naciendo en la vena porta derecha o la izquierda; esta variación vascular importante para los procedimientos radiológicos y quirúrgicos no altera el orden topográfico de los siete segmentos hepáticos (Fig. 4a y 4b) (Durand, 2016).

La fisura portal principal recibe esta denominación por su característica de dividir el lóbulo derecho del izquier- 
do; sin embargo cuando el pedículo segmentario $\mathrm{V}$ nace de la vena porta izquierda, es la fisura portal derecha la que separa el lóbulo derecho del izquierdo, tomando el papel principal, por esta razón proponemos la denominación de fisura "intermedia" (en vez de principal) acorde con su contenido venoso.

DURAND, L. C. A. Liver: the portal segment V. Int. J. Morphol., 35(1): 363-367, 2017.

SUMMARY: To determinate the characteristics of the segment $\mathrm{V}$ in the liver, we studied the intrahepatic vascular structure of 200 human livers taken from necropsies of males and females of different ages, from fetuses to octogenarians, all races. 150 livers were injected with acrylic in the portal vein, hepatic artery, biliary ducts, inferior cava vein and hepatic veins. We dissected 50 livers based on the knowledge obtained from the acrylic injected livers. We found that the segment $\mathrm{V}$ has only one branch, the largest of the seven portal segmental branches. After penetrates $2 \mathrm{~cm}$ in the hepatic parenchyma gives anterior branches, lateral branches, posterior branches and medial branches. This segmentary branch emerged from the right portal vein in $80 \%$ of cases and from the left portal vein in $20 \%$.

KEY WORDS: Portal segment V; Left portal vein; Right portal vein.

\section{REFERENCIAS BIBLIOGRÁFICAS}

Bianchi, H. F.; Algieri, R. D.; Sanjurjo, D.; Ottone, N. E. \& Fuentes, R. Multiple anatomical variations of the hepatic pedicle. Case report and clinical application. Int. J. Morphol., 32(3):782-5, 2014.

Chaib, E.; Ribeiro, M. A. Jr.; Saad, W. A. \& Gama-Rodrigues, J. The main hepatic anatomic variations for the purpose of split-liver transplantation. Transplant. Proc., 37(2):1063-6, 2005.

Couinaud, C. Lobes et segments hépatiques. Notes sur l'architecture anatomique et chirurgicale du foie. Presse Med., 62(33):709-12, 1954.

Couinaud, C. Surgical Anatomy of the Liver Revisited. Paris, Couinaud C., 1989.

Durand, L. C. A. Liver: seven hepatic segments. Int. J. Morphol., 34(4):1522-30, 2016

Fasel, J. H. \& Schenk, A. Concepts for liver segment classification: neither old ones nor new ones, but a comprehensive one. J. Clin. Imaging Sci., 3:48, 2013.

Healey, J. E. Jr. \& Schroy, P. C. Anatomy of the biliary ducts within the human liver; analysis of the prevailing pattern of branchings and the major variations of the biliary ducts. AMA Arch. Surg., 66(5):599-616, 1953.

Healey, J. E. Jr.; Schroy ,P. C. \& Sorensen, R. J. The intrahepatic distribution of the hepatic artery in man. J. Int. Coll. Surg., 20(2):133-48, 1953.

Hiatt, J. R.; Gabbay, J. \& Busuttil, R. W. Surgical anatomy of the hepatic arteries in 1000 cases. Ann. Surg., 220(1):50-2, 1994.

Hjortsjo, C. H. The topography of the intrahepatic duct systems. Acta Anat. (Basel), 11(4):599-615, 1951.

Hidayet, M. A.; Wahid, H. A.; Hidayet, A. M. \& Hidayet, N. M. A study of the intrahepatic vasculature in the human fetus, in the normal adult and in adults with portal cirrhosis. Surg. Gynecol. Obstet., 145(3):378-88, 1977.

Kimura, W.; Fukumoto, T.; Watanabe, T. \& Hirai, I. Variations in portal and hepatic vein branching of the liver. Yamagata Med. J., 33(2):11521, 2015.

Mitidieri, V. \& Loccisano, M. Dorsal sector of the liver. Rev. Argent. Anat. Online, 5(2):54-62, 2014.

Chanasong, R.; Putiwat, P.; Roboon, J. \& Sakulsak, N. Accessory hepatic artery arising from celiac trunk: an incidence in a Thai cadaver. Int. $J$. Morphol., 32(4):1136-9, 2014.

Terminology Committee of the IHPBA. Terminology of liver anatomy and resections. HPB Surg., 2:333-9, 2000.

Tolino, M. J.; Tartaglione, A. S.; Sturletti, C. D. \& García, M. I. Anatomy variations of biliary tree. Surgical implicance. Int. J. Morphol., 28(4):1235-40, 2010.

Dirección para correspondencia:

César Augusto Durand López

Cirujano del Hospital de Emergencias "José Casimiro Ulloa"

Av. Roosevelt 6355, Miraflores

Profesor de Anatomía Humana

Facultad de Medicina

Universidad Ricardo Palma

Lima

PERÚ

E-mail: cesaraugustoo1@gmail.com

Recibido: 23-04-2016

Aceptado:23-12-2016 